\title{
Child abuse associates with increased recruitment of perineuronal nets in the ventromedial prefrontal cortex: a possible implication of oligodendrocyte progenitor cells
}

\author{
Arnaud Tanti (iD ${ }^{1,2,5}{ }^{凶}$, Claudia Belliveau (iD ${ }^{1,3,5}$, Corina Nagy ${ }^{1}$, Malosree Maitra ${ }^{1,3}$, Fanny Denux ${ }^{1}$, Kelly Perlman (iD ${ }^{1,3}$, Frank Chen ${ }^{1}$, \\ Refilwe Mpai ${ }^{1,3}$, Candice Canonne ${ }^{1,3}$, Stéphanie Théberge ${ }^{1,3}$, Ashley McFarquhar ${ }^{1}$, Maria Antonietta Davoli ${ }^{1}$, Catherine Belzung (D) $^{2}$, \\ Gustavo Turecki (iD ${ }^{1,3,4}$ and Naguib Mechawar (D) ${ }^{1,3,4}$
}

(c) The Author(s) 2021

Child abuse (CA) is a strong predictor of psychopathologies and suicide, altering normal trajectories of brain development in areas closely linked to emotional responses such as the prefrontal cortex (PFC). Yet, the cellular underpinnings of these enduring effects are unclear. Childhood and adolescence are marked by the protracted formation of perineuronal nets (PNNs), which orchestrate the closure of developmental windows of cortical plasticity by regulating the functional integration of parvalbumin interneurons into neuronal circuits. Using well-characterized post-mortem brain samples, we show that a history of CA is specifically associated with increased densities and morphological complexity of WFL-labeled PNNs in the ventromedial PFC (BA11/12), possibly suggesting increased recruitment and maturation of PNNs. Through single-nucleus sequencing and fluorescent in situ hybridization, we found that the expression of canonical components of PNNs is enriched in oligodendrocyte progenitor cells (OPCs), and that they are upregulated in CA victims. These correlational findings suggest that early-life adversity may lead to persistent patterns of maladaptive behaviors by reducing the neuroplasticity of cortical circuits through the enhancement of developmental OPCmediated PNN formation.

Molecular Psychiatry (2022) 27:1552-1561; https://doi.org/10.1038/s41380-021-01372-y

\section{INTRODUCTION}

Child abuse (CA) has enduring effects on psychological development. Severe adversity during sensitive periods, during which personality traits, attachment patterns, cognitive functions, and emotional responses are shaped by environmental experiences, has a profound effect on the structural and functional organization of the brain [1].

At the cellular level, childhood and adolescence are marked by the protracted maturation of neural circuits, characterized by windows of heightened plasticity that precede the development of functional inhibitory connections and the balance of excitatory-inhibitory neurotransmission [2]. A major mechanism involved in this process is the recruitment of perineuronal nets (PNNs), a condensed form of extracellular matrix (ECM) forming most notably around parvalbumin-expressing $(\mathrm{PV}+)$ interneurons. PNNs are thought to gradually decrease heightened plasticity by stabilizing the integration and function of PV + cells into cortical networks and hindering the remodeling of these networks [3,4]. This has been notably linked to the persistence of long-term associations, including fear memories [5-7].

Evidence in rodents suggests that early-life stress associates with precocious functional maturation of $\mathrm{PV}+$ neurons and the early emergence of adult-like characteristics of fear and extinction learning [8], in addition to discrete changes in the immunoreactivity of inhibitory neuron markers and PNNs [9]. Taken together, these observations suggest that CA may alter the formation of PNNs.

We addressed this question using well-characterized postmortem samples from adult depressed suicides, who died during an episode of major depression with (DS-CA) or without (DS) a history of severe CA and from matched psychiatrically healthy individuals (CTRL). Standardized psychological autopsies were conducted to provide comprehensive post-mortem diagnosis and retrieve various dimensions of childhood experience, including history and severity of CA. We focused on the ventromedial prefrontal cortex (vmPFC), encompassing Brodmann areas 11 and 12 in our study, a brain area closely linked to emotional learning, and which is structurally and functionally altered in individuals with a history of CA [1,10-13].

\section{MATERIALS AND METHODS}

Human post-mortem brain samples

Brain samples were obtained from the Douglas-Bell Canada Brain Bank (Montreal, Canada). Phenotypic information was retrieved through standardized psychological autopsies, in collaboration with the Quebec

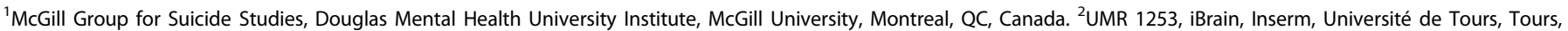

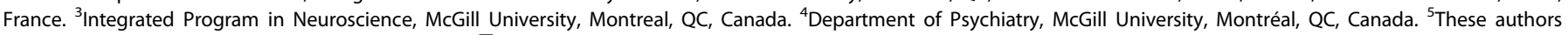
contributed equally: Arnaud Tanti, Claudia Belliveau. ${ }^{凶}$ email: arnaud.tanti@inserm.fr; naguib.mechawar@mcgill.ca
} 
Table 1. Group characteristics.

\begin{tabular}{|c|c|c|c|}
\hline & CTRL & DS & DS-CA \\
\hline$N$ & 11 & 16 & 12 \\
\hline Axis 1 diagnosis & 0 & MDD (14); DD-NOS (2) & MDD (11); DD-NOS (1) \\
\hline Age (years) $(P=0.38)$ & $43.18 \pm 7.11$ & $46.63 \pm 3.48$ & $37.75 \pm 3.10$ \\
\hline $\operatorname{Sex}(M / F)$ & $9 / 2$ & $14 / 2$ & $9 / 3$ \\
\hline PMI (h) $(P=0.74)$ & $35.95 \pm 7.15$ & $45.95 \pm 8.06$ & $40.92 \pm 6.68$ \\
\hline Tissue $\mathrm{pH}(P=0.42)$ & $6.40 \pm 0.09$ & $6.52 \pm 0.07$ & $6.56 \pm 0.08$ \\
\hline $\begin{array}{l}\text { Substance } \\
\text { dependence }\end{array}$ & 0 & 6 & 5 \\
\hline Medication & 0 & $\begin{array}{l}\text { SSRI (4); SNRI (1); TCA (1); Benzodiazepines (3); } \\
\text { Antipsychotics (2); Antimanic (1) }\end{array}$ & $\begin{array}{l}\text { SSRI (2); Benzodiazepines (2); Antipsychotics } \\
\text { (1); Antimanic (1) }\end{array}$ \\
\hline
\end{tabular}

Data represent mean \pm SEM. $P$-values generated with one-way ANOVAs.

$D D$-NOS depressive disorder not otherwise specified, MDD major depressive disorder, PMI post-mortem interval, SNRI selective norepinephrine reuptake inhibitor, SSRI selective serotonin reuptake inhibitor, TCA tricyclic antidepressant.

Coroner's Office and with informed consent from the next of kin. Presence of any or suspected neurological/neurodegenerative disorder signaled in clinical files constituted an exclusion criterion. Cases and controls are defined with the support of medical charts and Coroner records. Proxybased interviews with one or more informants best acquainted with the deceased are supplemented with information from archival material obtained from hospitals, Coroner's office, and social services. Clinical vignettes are then produced and assessed by a panel of clinicians to generate Diagnostic and Statistical Manual of Mental Disorders (DSM-IV) diagnostic criteria, providing sociodemographic characteristics, social developmental history, DSM-IV axis I diagnostic information, and behavioral traits-information that is obtained through different adapted questionnaires. Toxicological assessments and medication prescription are also obtained. As described previously [14], characterization of early-life histories was based on adapted Childhood Experience of Care and Abuse interviews assessing experiences of sexual and physical abuse, as well as neglect [15], and for which scores from siblings are highly concordant [16]. We considered as severe early-life adversity (ELA) reports of non-random major physical and/or sexual abuse during childhood (up to 15 years). Only cases with the maximum severity ratings of 1 and 2 were included. This information was then complemented with medical charts and Coroner records. Because of this narrow selection criterion, it was not possible to stratify different types of abuse within the sample.

Group characteristics are described in Table 1. Correlations between covariates (age, post-mortem interval (PMI), pH, substance dependence, and medication) and the variables measured in our study are presented in Supplementary Table 1.

\section{Tissue dissections}

Dissections were performed by expert brain bank staff on fresh-frozen 0.5 $\mathrm{cm}$-thick coronal sections with the guidance of a human brain atlas [17]. vmPFC samples were dissected in sections equivalent to plate 3 (approximately $-48 \mathrm{~mm}$ from the center of the anterior commissure) of this atlas, corresponding to Brodmann areas 11 and 12. Samples were either kept frozen or fixed overnight in $10 \%$ formalin until processed for in situ hybridization or immunofluorescence, respectively. Samples used for PV immunohistochemistry were stored long term in 10\% formalin until processed.

\section{Immunostaining}

Frozen tissue blocks were fixed in $10 \%$ neutral buffered formalin overnight at $4{ }^{\circ} \mathrm{C}$, rinsed in phosphate-buffered saline (PBS), and kept in $20 \%$ sucrose/ PBS solution until serially sectioned at $40 \mu \mathrm{m}$ on a cryostat. Free-floating sections were rinsed in PBS and then incubated overnight at $4{ }^{\circ} \mathrm{C}$ under constant agitation with the antibody (mouse anti-NeuN (Millipore, $1: 500$, MAB377), goat anti-Versican (R\&D, $1: 100, A F 3054)$ ), or lectin (biotinylated Wisteria Floribunda Lectin (WFL), Vector Laboratories, $1: 2500, \mathrm{~B}-1355)$ of interest diluted in a blocking solution of $\mathrm{PBS} / 0.2 \%$ Triton- $\mathrm{X} / 2 \%$ normal donkey serum. Sections were then rinsed and incubated for $2 \mathrm{~h}$ at room temperature with the appropriate fluorophore-conjugated secondary antibody (Alexa-488 anti-Mouse (Jackson ImmunoResearch, 1:500) for NeuN, Dylight-594 anti-goat (Jackson ImmunoResearch, 1:500) for VCAN, or Cy3-conjugated Streptavidin (Jackson ImmunoResearch, 016-160-084; $1: 500)$ for the detection of PNNs, and diluted in the same blocking solution as the primary incubation. Next, sections were rinsed and endogenous autofluorescence from lipofuscin and cellular debris was quenched with Trueblack (Biotium), omitted for tissues used for intensity measurements. Sections were mounted on Superfrost charged slides and coverslipped with Vectashield mounting medium (Vector Laboratories, $\mathrm{H}$ 1800).

Whole vmPFC sections were scanned on a Zeiss Axio Imager M2 microscope equipped with a motorized stage and Axiocam MRm camera at $\times 20$. The ImageJ [18] software (NIH) Cell Counter plugin was used by a blinded researcher to manually count PNNs. An average of four sections per subject was used. Cortical layers were delineated based on NeuN + cell distribution and morphology, and the number of PNNs and the area of each layer were measured, allowing to generate PNN density values ( $\mathrm{n}$ / $\mathrm{mm}^{2}$ ). Densities were obtained by averaging by subject the density of PNNs per layer and section, and then averaging subjects' densities to yield group means.

For PV immunohistochemistry, tissue blocks stored in $10 \%$ formalin were first transferred to $30 \%$ sucrose/PBS. Once sunk, blocks were flash frozen in isopentane and kept at $-80^{\circ} \mathrm{C}$ until embedded and serially sectioned on a sliding microtome $(40 \mu \mathrm{m})$. Prior to immunohistochemical staining, tissues underwent antigen retrieval by incubating for $15 \mathrm{~min}$ in hot $10 \mathrm{mM}$ sodium citrate buffer pH 6.0 (Sigma catalog number S-4641). Sections were rinsed in PBS and incubated in $3 \% \mathrm{H}_{2} \mathrm{O}_{2} / \mathrm{PBS}$ for $15 \mathrm{~min}$. After being rinsed, sections were incubated overnight at $4{ }^{\circ} \mathrm{C}$ under constant agitation with a mouse anti-Parvalbumin antibody (Swant, $1: 500$, PV235) diluted in a blocking solution of $\mathrm{PBS} / 0.2 \%$ Triton-X/2\% normal horse serum. Sections were then rinsed and incubated for $2 \mathrm{~h}$ at room temperature in biotinylated horse anti-mouse antibody $(1: 500$, Vector Laboratories, Inc., BA-2001, Burlington, ON, Canada). Then, sections were incubated in the avidin-biotin complex (ABC Kit, Vectastain Elite, Vector Laboratories, Inc., Burlington, ON, Canada) for $30 \mathrm{~min}$ at room temperature. Labeling was revealed with the diaminobenzidine (DAB) kit (Vector Laboratories, Inc., Burlington, ON, Canada), then sections were rinsed and mounted on Superfrost charged glass slides, dehydrated, and coverslipped with Permount (Fisher Scientific, Inc., Pittsburgh, PA, USA). Immunohistological controls were performed by omitting primary antibodies. After a first round of imaging, the coverslips were removed and samples were counterstained with cresyl violet to differentiate cortical layers and imaged a second time. Image acquisition was performed on an Olympus VS120 Slide Scanner at $\times 10$. Image analysis was performed in QuPath [19] ( $v 0.1 .2$ ). Automatic cell detection was used to detect PV+ cells. DAB images were overlaid on the cresyl-counterstained image using the function Interactive Image Alignment - which allowed a blinded researcher to delineate the cortical layers based on cresyl violet-stained cells. Densities were calculated by cortical layer.

\section{Fluorescent in situ hybridization}

Frozen vmPFC blocks were cut serially with a cryostat and $10 \mu \mathrm{m}$-thick sections were collected on Superfrost charged slides. In situ hybridization was performed using Advanced Cell Diagnostics RNAscope ${ }^{\circledast}$ probes and reagents following the manufacturer's instructions. Sections were first fixed 
in cold $10 \%$ neutral buffered formalin for $15 \mathrm{~min}$, dehydrated by increasing gradient of ethanol baths, and air dried for $5 \mathrm{~min}$. Endogenous peroxidase activity was quenched with hydrogen peroxide for $10 \mathrm{~min}$ followed by protease digestion for $30 \mathrm{~min}$ at room temperature (omitted for samples undergoing subsequent WFL staining). The following probes were then hybridized for $2 \mathrm{~h}$ at $40^{\circ} \mathrm{C}$ in a humidity-controlled oven: Hs-PVALB (catalog number 422181), Hs-VCAN (catalog number 430071-C2), Hs-PDGFRA (catalog number 604481-C3), Hs-TNR (catalog number 525811), Hs-PTPRZ1 (catalog number 584781-C2), Hs-SLC17A7 (catalog number 415611), and Hs-GAD1 (catalog number 573061-C3). Amplifiers were added using the proprietary AMP reagents and the signal visualized through probe-specific HRP-based detection by tyramide signal amplification (TSA) with Opal dyes (Opal 520, Opal 570, or Opal 690; Perkin Elmer) diluted 1:700. Slides were then coverslipped with Vectashield mounting medium with 4',6-diamidino-2-phenylindole (DAPI) for nuclear staining (Vector Laboratories) and kept at $4{ }^{\circ} \mathrm{C}$ until imaging. Both positive and negative controls provided by the supplier (ACDbio) were used on separate sections to confirm signal specificity. For immunohistochemical staining of PNNs following PVALB, GAD1 (glutamate decarboxylase 1), or SLC17A7 (vesicular glutamate transporter 1) in situ hybridization, slides were rinsed in PBS, incubated for $30 \mathrm{~min}$ at room temperature with biotinylated WFL, followed by 488 conjugated Streptavidin for $30 \mathrm{~min}$ prior to coverslipping. To better define the cellular identity of neuronal populations surrounded by WLF-labeled PNNs, TrueBlack was used to remove endogenous autofluorescence from lipofuscin and cellular debris.

\section{Cellular identity and ratios of each cell type surrounded by WFL-labeled PNNs}

Image analysis was performed in QuPath ( $v$ 0.2.3). Each subject had two sections stained with various cellular markers: DAPI, PVALB, SLC17A7, GAD1, and WFL. To identify the population of cells covered by PNNs and calculate the percentage of each cell type that is covered by a net; a blinded researcher manually identified PNNs and categorized each nucleus within a region of interest (spanning layers $\mathrm{III}-\mathrm{VI}$ ) dependent on the presence of canonical cellular markers. A total of 3145 PNNs were classified and a total of 18,600 SLC17A7+, 2209 GAD1+/PVALB + and 8659 GAD1+/PVALB - cells were classified.

A replication experiment was conducted for the proportions of PVALB+ cells enwrapped by WFL-labeled PNNs, which were determined in a single section with an average of 55 PVALB + cells per subject imaged under a $\times 20$ objective through vmPFC layers IV-V.

\section{Imaging and analysis of in situ RNA expression in OPCs}

Image acquisitions was performed on a FV1200 laser scanning confocal microscope (FV1200) equipped with a motorized stage. For each experiment and subject, six to ten stack images were taken to capture at least 20 oligodendrocyte progenitor cells (OPCs) (PDGFRA+) per subject. Images were taken using a $\times 60$ objective $(N A=1.42)$ with an $X Y$ pixel width of $\sim 0.25 \mu \mathrm{m}$ and Z-spacing of $0.5 \mu \mathrm{m}$. Laser power and detection parameters were kept consistent between subjects for each set of experiment. As TSA amplification with Opal dyes yields a high signal-tonoise ratio, parameters were optimized so that autofluorescence from lipofuscin and cellular debris was filtered out. OPCs were defined by bright clustered puncta-like PDGFRA signal present within the nucleus of the cells. Using ImageJ, stacks were first converted to Z-projections, and for each image the nuclei of OPCs were manually contoured based on DAPI expression. Expression of versican (VCAN), tenascin-R (TNR), or phosphacan (PTPRZ1) in OPCs was quantified using the area fraction, whereby for each probe the signal was first manually thresholded by a blinded researcher and then the fraction of the contoured nucleus area covered by signal was measured for each OPC. Area fraction was the preferred measure to reflect RNA expression, as punctate labeling generated by fluorescence in situ hybridization (FISH) often aggregates into clusters that cannot readily be dissociated into single dots or molecules.

\section{Intensity, area, and distance measurements}

For each subject, $\sim 15 \mathrm{Z}$-stacks $(0.26 \mu \mathrm{m} \mathrm{Z}$-spacing) spanning all layers of the vmPFC were acquired at $\times 40$ magnification on an Olympus FV1200 laser scanning confocal microscope. Images for intensity measurement were all acquired at the same laser strength and voltage to avoid imaging differences in intensity or over-exposure. PNNs were traced manually with ImageJ by a blinded researcher using maximum intensity projections generated from each stack. All the PNNs that were observed were traced as long as their whole morphology was in the field of view. For each PNN, the mean pixel value of adjacent background was subtracted to the mean pixel value of the contoured soma of the PNN, yielding the mean corrected fluorescence intensity (arbitrary units). To infer on their morphological complexity, we measured the area covered by each contoured PNN, including soma and ramifications extending over proximal dendrites.

To quantify closest distance between OPCs and PV+ cells, low magnification $(\times 10)$ images of PDGFRA and PVALB FISH sections were taken by a blinded researcher along layers IV and $V$ of the VmPFC, using the granular layer IV as a visual reference. For each PDGFRA+ cell in the field of view, the distance to the nearest $P V A L B+$ cell was measured using the measure tool in ImageJ. An average of 90 OPCs per group were quantified.

\section{OPC density measurements}

Image acquisition was performed on an Olympus VS120 Slide Scanner at $\times 20$. Image analysis was performed in QuPath $(v 0.2 .3)$ by a blinded researcher. Automatic cell detection was used to detect DAPI nuclei. Then, an object classifier was trained on five training images from five different subjects. Cells were deemed PDGFRA+ based on the mean intensity of PDGFRA staining compared to the mean staining of a background channel. In total, 21 subjects were included $(C T R L=6, D S=7$, and $D S-C A=8)$ in this analysis.

\section{Cell-type-specific expression of PNN canonical components using single-nucleus sequencing}

Cell-type-specific expression of canonical components of PNNs was explored using a single-nucleus RNA sequencing (snRNA-seq) data set from the human dorsolateral PFC (BA9) previously generated by our group [20], for which methodology is extensively described in this published resource. Average expression for each PNN component in each cell type was calculated by weighting the expression values (normalized transcript counts) of each cluster by the size (number of nuclei) of the cluster. Weighted average expression values are displayed in a heatmap, scaled by row (i.e., gene). The color bar therefore represents the expression values as $z$-scores, with darker colors indicating higher expression.

\section{Statistical analyses}

Independently of $\mathrm{PMI}, \mathrm{pH}$, and approach used, the quality of post-mortem samples is notoriously variable and is influenced by tissue degradation, quality of fixation, and other artefacts. Only samples that showed reliable labeling were included in the different experiments without prior knowledge about group affiliation. Analyses were performed on Statistica version 12 (StatSoft) and Prism version 6 (GraphPad Software). Distribution and homogeneity of variances were assessed with Shapiro-Wilk and Levene's tests, respectively. PNN densities were analyzed using a mixedeffects model, using layer and group as fixed factors, followed by Tukey's honestly significant difference test for corrected post hoc comparisons. For all other variables (WFL intensity, WFL area per PNN, PNN + /PVALB ratios, RNA expression in OPCs and distance of OPCs from PVALB+ cells) group effects were detected using one-way ANOVAs or Kruskal-Wallis test followed by Tukey's honestly significant difference or Dunn's test, respectively. Linear regressions and Spearman's correlation were used to address the relationship between dependent variables and covariates (age, $\mathrm{PMI}$ and $\mathrm{pH}$, medication, and substance dependence) (Supplementary Table 1). Statistical tests were two-sided. Significance threshold was set at 0.05 and all data presented represent mean \pm SEM.

\section{RESULTS}

PNN densities, visualized by WFL labeling and NeuN immunostaining (Fig. 1A), were markedly higher through layers III-VI of vmPFC (BA11/12) samples from individuals with a history of CA compared to controls and depressed suicides with no history of CA (Fig. 1B). Although the recruitment of PNNs is developmentally regulated, we did not find any correlation between age and densities of PNNs (Supplementary Table 1), perhaps suggesting that PNN recruitment may already have reached a plateau in our cohort [21]. Likewise, controlling for age did not affect our results (analysis of covariance (ANCOVA) with group as fixed factor and age as covariate; group effect: $F(2,35)=11.230, P<0.001)$. To investigate whether $C A$ also associates with maturational or 
A

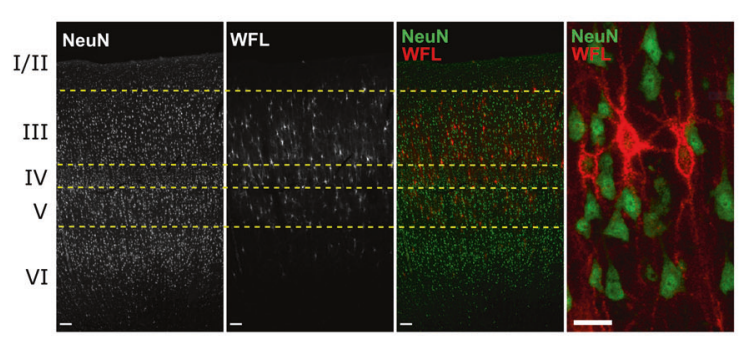

C
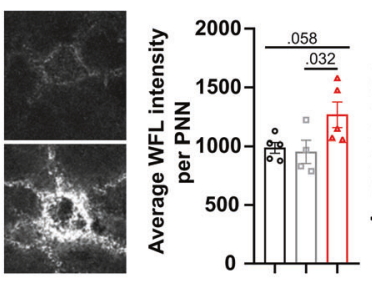

D

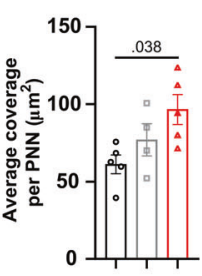

F

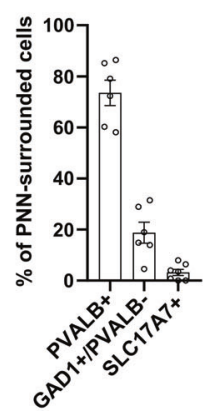

G
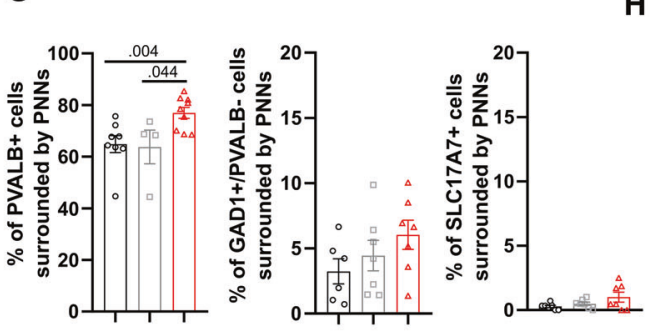

H

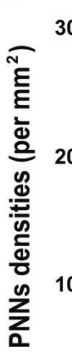

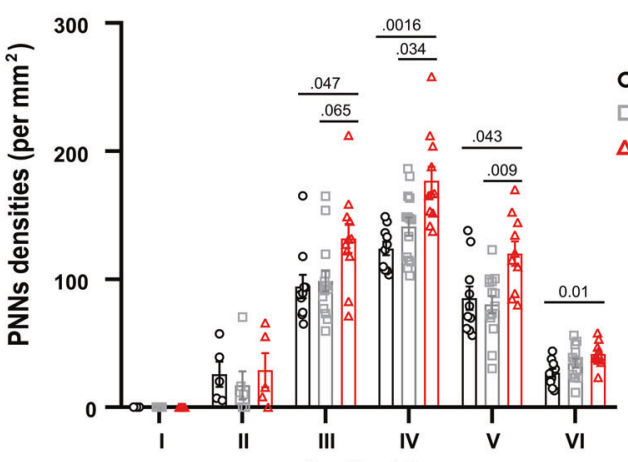

E
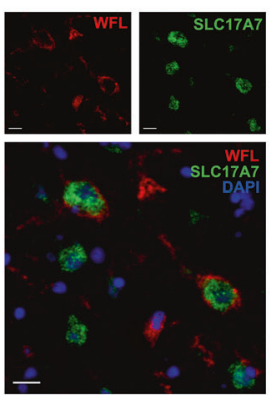

Cortical layer

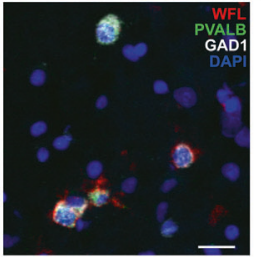

$\square \mathrm{DS}$

$\triangle$ DS-CA
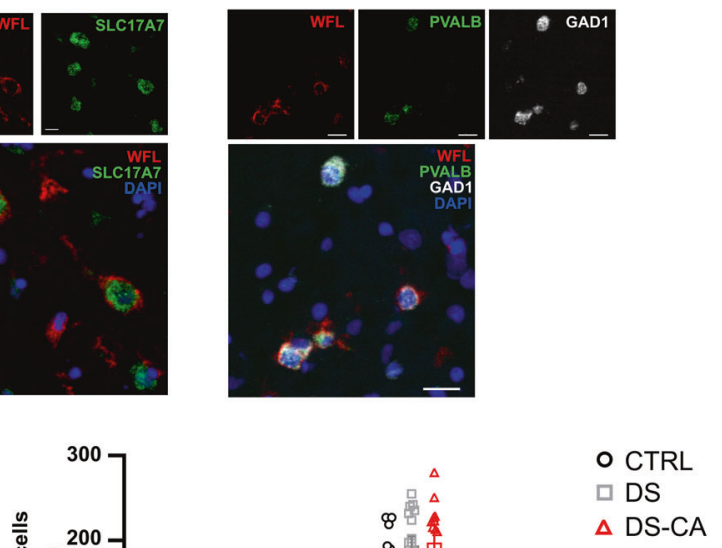

$\square \mathrm{DS}$

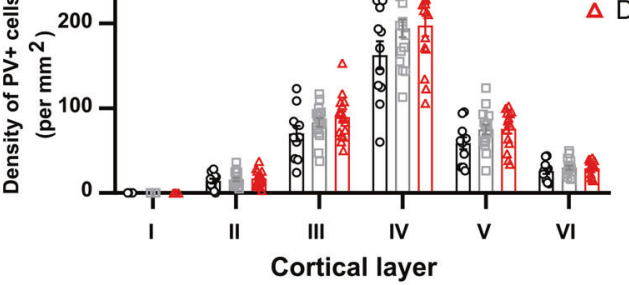

Fig. 1 Increased density, morphological complexity and recruitment of PNNs around parvalbumin neurons in the vmPFC of depressed suicides with a history of child abuse. A Representative images of PNNs labeled with WFL and their distribution throughout human vmPFC cortical layers. Scale bars $=100$ and $20 \mu \mathrm{m}$ (high-magnification panel). B Depressed suicides with a history of child abuse (DS-CA, $N=11)$ have significantly higher PNN densities compared to controls $(C T R L, N=10)$ and depressed suicides without history of child abuse $(D S, N=14)$ (group effect: $F(2,32)=7.029, P=0.0029$; layer effect: $F(3.395,78.09)=194.2, P<0.0001$; layer $\times$ group: $F(10,115)=2.07, P=0.0029$, followed by Tukey's multiple comparison test). C Representative images of a low (top) and high (bottom) intensity PNN in the vmPFC. PNNs from DS-CA subjects $(N=5)$ showed higher average WFL intensity (arbitrary units) compared to CTRLs $(N=5)$ or DS ( $N=4)$ (Kruskal-Wallis ANOVA: H(2, $14)=5.57, P=0.049$, followed by Dunn's test). D PNNs from DS-CA subjects $(N=5)$ showed higher complexity (area covered by PNNs) compared to CTRLs $(N=5)$ or DS $(N=4)$ (Kruskal-Wallis ANOVA: $\mathrm{H}(2,14)=6.223, P=0.034$, followed by Dunn's test). E Left: representative images of in situ hybridization for SLC17A7 (green) followed by WFL labeling (red). Nuclei were stained with DAPI (blue); right: representative images of in situ hybridization for PVALB (green) and GAD1 (white) followed by WFL labeling (red). Nuclei were stained with DAPI (blue). Scale bars $=20 \mu \mathrm{m}$. F Proportions of WFL-labeled PNNs expressing PVALB (PV+ neurons), GAD1 but not PVALB (other inhibitory neurons), and SLC17A7 (excitatory neurons). G DS-CA ( $N=9$ ) subjects have higher ratios of $P V A L B+$ cells surrounded by PNNs compared to CTRLs $(N=8)$ and DS subjects $(N=4)$ (Kruskal-Wallis ANOVA $\mathrm{H}(2,21)=9.45, P=0.0037$, followed by Dunn's test), but not of GAD1+/PVALB- cells (Krukal-Wallis ANOVA $\mathrm{H}(2,20)=3.28, P=0.2)$ nor SLC17A7+ cells (Kruskal-Wallis ANOVA, $\mathrm{H}(2,21)=2.58, P=0.29)$. G Densities of PV + cells assessed by immunohistology. No change between groups was observed (group effect: $P=0.132$; layer effect: $P<0.0001 ;$ group $\times$ layer: $P=0.083$ ).

morphological changes of PNNs, we compared the intensity of WFL staining between groups (Fig. 1C) as an indication of their maturity, as well as the area covered by individual PNNs as an indicator of their morphological complexity (Fig. 1D). CA was both associated with higher intensity of WFL staining per PNN (Fig. 1C) and cells were more extensively covered by PNNs (Fig. 1D), suggesting overall that CA may precipitate the maturation and the recruitment of PNNs.

PNNs have been most extensively described around PV+ cells, but are also found around other neuronal types [22]. We first wanted to specify the identity of cells covered by PNNs in the human vmPFC. We measured the ratios of PNNs surrounding either PV+ cells, glutamatergic neurons, and other interneurons in a subset of control, psychiatrically healthy subjects (Fig. 1E, F). As PV antigenicity is particularly susceptible to freezing and lost altogether in samples snap frozen prior to fixation, we developed an approach to combine FISH and immunofluorescence to visualize PVALB-expressing cells and WFL + PNNs in frozen samples. Similarly, glutamatergic neurons and other subtypes of interneurons were visualized with FISH using probes against SLC17A7 and GAD1, respectively (Fig. 1E). The majority of cells covered by WFL staining were PVALB+ ( 74\%), followed by 
GAD1+/PVALB - cells ( 23\%), indicating that a small fraction of PNNs is likely surrounding other subtypes of interneurons (Fig. 1F). As previously reported $[23,24]$, some PNNs stained with WFL were also found to surround glutamatergic neurons (SLC17A7+), although only a very small fraction of them ( 3\%, Fig. 1F).

To clarify the cellular specificity of the observed increase in PNNs recruitment, we next quantified the ratios of PVALB+, SLC17A7+, and GAD1+/PVALB - cells surrounded by WFL+PNNs. Here, $\sim 65 \%$ of PVALB + cells were surrounded by PNNs (Fig. 1G), in line with previous observations [25], whereas only a small proportion of GAD1 +/PVALB - and SLC17A7+ cells were covered by PNNs (Fig. 1G). Interestingly, ratios of cells covered by WFL-labeled PNNs showed a positive correlation with age (Supplementary Table 1), suggesting an increased recruitment of PNNs with age regardless of cell type. Importantly, samples from DS-CA individuals displayed a robust increase in the percentage of PVALB + cells surrounded by PNNs compared to DS and CTRL samples (Fig. 1G), whereas no change in the proportion of other cell types covered by PNNs was found between group. Controlling for age as a covariate did not change the outcome of these group comparisons (\%PVALB+/PNN+ cells: $\mathrm{F}(2,15)$ $=4.08, P=0.047 ; \% G A D 1+/ P V A L B-/ P N N+$ cells: $\mathrm{F}(2,15)=0.237, P=$ 0.793; \%SLC17A7+/PNN+ cells: $\mathrm{F}(2,15)=1.430, P=0.281)$.

Finally, we addressed whether the increased densities of PNNs and higher ratios of PV + cells surrounded by PNNs observed in DS-CA subjects could be linked to changes in the number of PV+ cells in the vmPFC and found no evidence of altered PV+ cell densities between groups (Fig. 1H). Of note, PV+ cell densities were inversely correlated with age (Supplementary Fig. 1), but controlling for this factor did not change the outcome of group comparisons (ANCOVA with group as fixed factor and age as covariate: group effect, $P=0.251$; age effect, $P=0.007$ ).

Altogether, these results suggest that a history of $C A$ in depressed suicides is associated with increased recruitment and maturation/morphological complexity of PNNs around PV+ neurons, rather than changes in cell populations.

We then sought to indirectly explore the molecular underpinnings of this phenomenon and reasoned that increased recruitment of PNNs associated with CA should translate or be induced by changes in the molecular programs controlling PNN assembly. Our understanding of these transcriptional programs is scarce, hindered by the fact that several known molecules participating in PNN recruitment are released non-locally and by different cell types, implying a complex cellular crosstalk orchestrating PNN assembly. To gain insight into how, in humans, different cell types contribute to the synthesis of canonical components of PNNs, we explored a single-nucleus sequencing data set previously generated by our group in the human dorsolateral PFC [20] (Brodmann area 9) and screened their expression across eight major cell types. The main canonical components of PNNs, namely aggrecan $(A C A N)$, neurocan $(N C A N)$, versican (VCAN), phosphacan (PTPRZ1), brevican (BCAN), and tenascin-R $(T N R)$, were highly enriched in OPCs, in particular VCAN, PTPRZ1, BCAN, and TNR (fold change of 140,37, 7.9, and 22.9, respectively, between gene expression in OPCs vs. PV+ cells) (Fig. 2A and Supplementary Fig. 1). As this data set originates from the dIPFC and region-specific patterns of ECM-related gene expression could exist, this was further validated using FISH (Fig. 2B, C) in the vmPFC for VCAN and PTPRZ1, as they showed the strongest expression in OPCs and are two major signature genes in late OPCs [26]. We found that in the vmPFC, cells expressing these genes are almost all PDGFRA+OPCs $(97.9 \%$ of VCANexpressing cells were co-expressing PDGFRA, and 92\% of PTPRZ1expressing cells were co-expressing PDGFRA) (Fig. 2D). Interestingly, despite that VCAN gene expression was restricted to OPCs, immunolabeling of the versican protein showed a characteristic pattern of PNNs and an overlap with WFL-labeled PNNs (Fig. 2E), suggesting overall that OPCs could be potent regulators of PNN formation.
In support of a possible involvement of OPCs in mediating CArelated changes in PNNs, the expression of VCAN, PTPRZ1, and TNR was upregulated in OPCs of CA victims (Fig. $2 \mathrm{~F}-\mathrm{H}$ ), and both the expression of PTPRZ1 and TNR in OPCs correlated with WFLlabeled PNNs densities regardless of group (Fig. 2l, J).

OPCs were on occasion directly juxtaposed to PVALB+ cells (Fig. 2B), as previously reported in rodents [27]. Interestingly, OPC proximity to PVALB + cells modestly correlated with PNN density (Fig. $2 \mathrm{~L}, R^{2}=0.36, P=0.024$ ) and was increased in individuals with a history of CA (Fig. 2M), further suggesting an interplay between these two cell types. To clarify whether these changes could be linked or associated with changes in cell numbers, we compared the density of OPCs between groups. Interestingly OPC densities showed a marked decrease with age (Supplementary Table 1), but no difference between groups were found (Fig. 2N), even after controlling for this factor (ANCOVA, with group as fixed factor and age as covariate: group effect, $P=0.13$; age affect, $P=0.001$ ).

\section{DISCUSSION}

Overall, our results suggest that a history of CA may associate with increased recruitment and maturation of PNNs, as well as an upregulation of their canonical components by OPCs, a cell type that likely plays a key role in the cellular crosstalk that orchestrates PNN formation.

Although, to our knowledge, this is the first evidence in humans that ELA affects the recruitment of PNNs, recent studies in animals have approached this question. Guadagno et al. [28] found that in the limited bedding paradigm, adolescent pups have increased densities of PNNs in the amygdala. Murthy et al. [9] showed that in the ventral dentate gyrus, maternal separation combined with early-weaning, another model of early-life stress mimicking some aspects of adversity, led to an increase in PNN intensity around parvalbumin-positive interneurons with no change in PNN density. Importantly, these effects were present in adults, suggesting a long-lasting impact of ELA on PV+ cell function and PNN remodeling. Gildawie et al. [29] also recently reported that in the prelimbic cortex, maternal separation in rats increased the intensity of PNNs surrounding PV + neurons, an effect only observed in adulthood. This suggests that changes in PNN integrity and maturation following ELA could possibly be protracted and develop over time. Although we cannot address this question with our post-mortem design, our results show at the very least that changes in PNN integrity in victims of CA are observable at an adult age. Few studies have so far been conducted on this topic, however, with one of them showing a decrease in the intensity of WFL fluorescent labeling in both the PFC and hippocampal CA1 following an early-life sub-chronic variable stress paradigm [30]. Clearly, more work is needed to better characterize the effects of ELA on PNNs integrity, particularly in light of the multiple paradigms used in animal studies.

Despite the fact that much of the literature has focused on the influence of PNNs on PV+ cell physiology, it is important to note that PNNs are not exclusively present around PV + neurons $[22,23,31]$. Although our data indicate that in the vmPFC PVsurrounding PNNs are the majority, a portion of them were identified around other GABAergic neurons, as well as around a small fraction of excitatory neurons. Our data suggest that CA associates with a selective increase in the recruitment of PNNs around $\mathrm{PV}+$ neurons, considering that the higher percentage of cells surrounded by WFL-labeled PNNs was only found for PV+ cells, but not for other cell types. However, we cannot exclude that given the smaller pool of non-PV cells surrounded by WFL-labeled PNNs, we were unable to detect such effects in our design. It is also likely that the sole use of WFL immunostaining to detect PNNs may be a limitation to fully understand their distribution. It is becoming increasingly clear that PNNs vary in molecular 

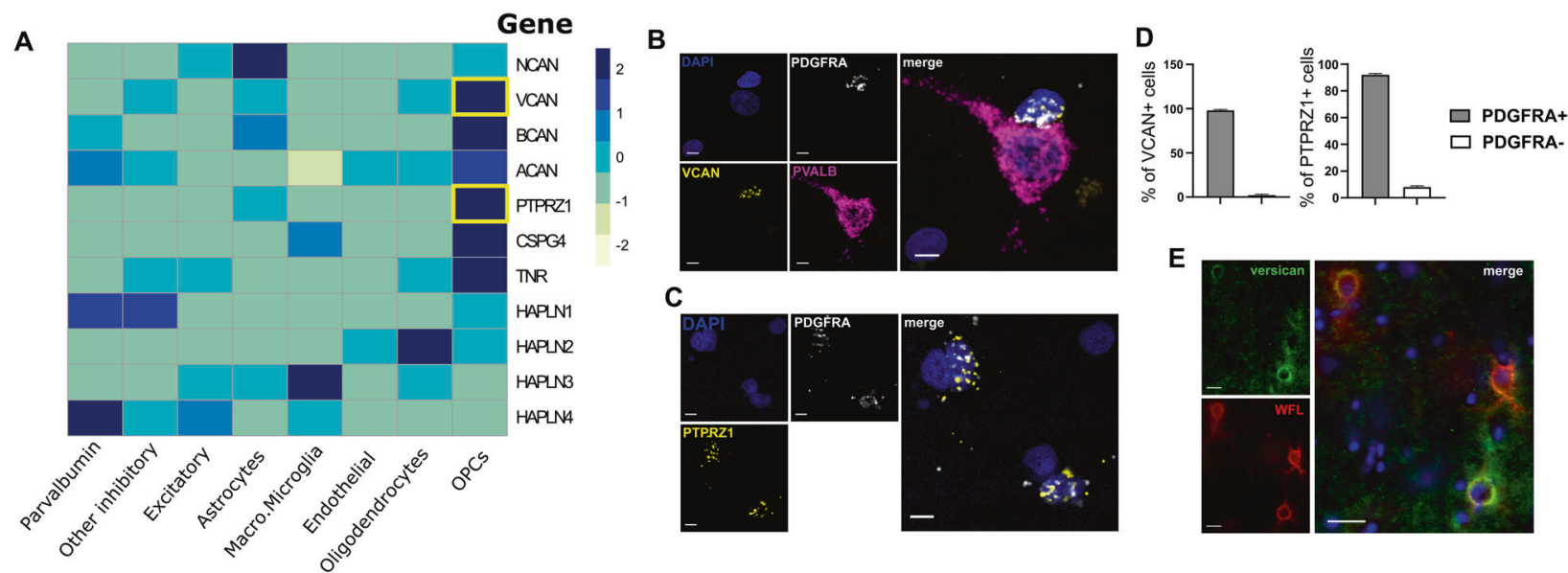

\section{Cell type}

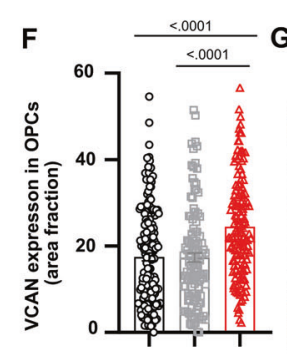

K

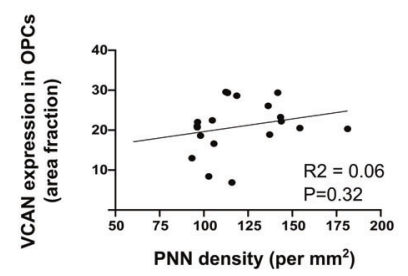

H

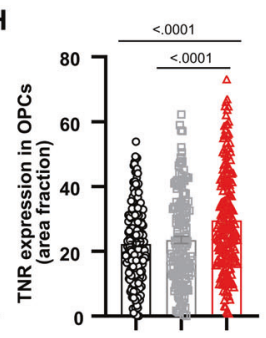

L

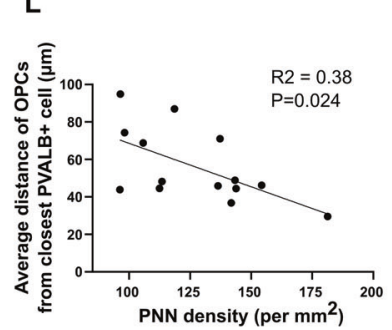

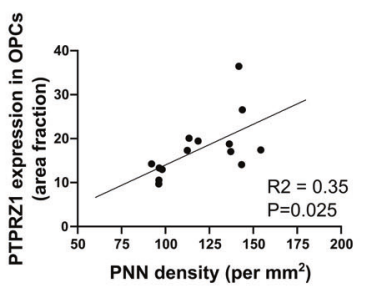

M

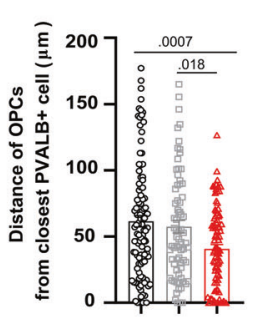

N
J
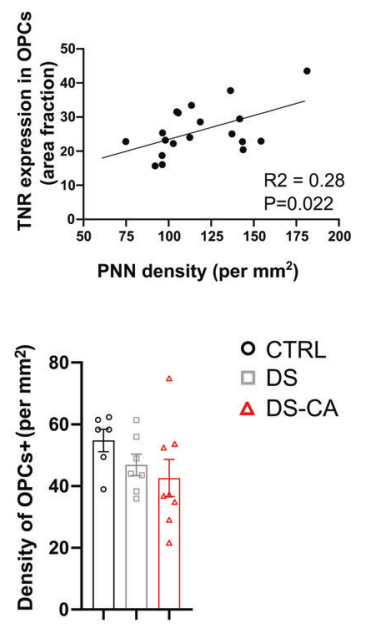

○ CTRL

$\square \mathrm{DS}$

$\triangle$ DS-CA

Fig. 2 Gene expression of canonical PNN components is enriched in OPCs and upregulated in depressed suicides with a history of child abuse. A Expression of canonical components of PNNs according to cell type, derived from single-nucleus RNA sequencing of 34 human dIPFC (BA9) samples [20]. OPCs consistently express higher levels of most of these components compared to other cell types. B Representative images of FISH validation of VCAN (Versican, yellow) expression in OPCs (PDGFRA+ cells, white). Note the VCAN-expressing OPC juxtaposed to a PVALB + (magenta) cell. Nuclei were counterstained with DAPI (blue). Scale bar $=5 \mu \mathrm{m}$. C Representative images of FISH validation of PTPRZ1 (Phosphacan, yellow) expression in OPCs (PDGFRA+ cells, white). Nuclei were counterstained with DAPI (blue). Scale bar $=5 \mu \mathrm{m}$. D Both VCAN (left) and PTPRZ1 (right) expression is highly enriched in OPCs, with $97.8 \%$ of VCAN+ cells $(N=225)$ co-expressing PDGFRA and $91.8 \%$ of PTPRZ1 + cells $(N=281)$ co-expressing PDGFRA. E Representative image of versican (green) immunolabeling. Despite enrichment of VCAN gene in OPCs, the versican protein shows a characteristic PNN staining pattern and colocalized with WFL (red). Nuclei were counterstained with DAPI (blue). Scale bar $=25 \mu \mathrm{m}$. F The average expression of VCAN in OPCs was significantly higher in DS-CA subjects ( $N=139$ cells, 7 subjects) compared to CTRLs ( $N=160$ cells, 8 subjects) and DS ( $N=119$ cells, 6 subjects) (one-way ANOVA F $(2,415)=17.25, P<0.0001$, followed by Tukey's multiple comparison test). G The average expression of PTPRZ1 in OPCs was significantly higher in DS-CA subjects ( $N=63$ cells, 4 subjects) compared to CTRLs ( $N=117$ cells, 6 subjects) and DS ( $N=81$ cells, 5 subjects) (one-way ANOVA $F(2,258)=31.65, P<0.0001$, followed by Tukey's multiple comparison test). $\mathbf{H}$ The average expression of TNR in OPCs was significantly higher in DS-CA subjects ( $N=200$ cells, 8 subjects) compared to CTRLs ( $N=207$ cells, 7 subjects) and DS ( $N=160$ cells, 5 subjects) (one-way ANOVA, $F(2,564)=18.69, P<0.0001$, followed by Tukey's multiple comparison test). Both PTPRZ1 (I) and TNR (J), but not VCAN (K) average expression in OPCs modestly correlated with PNNs densities $\left(R^{2}=0.35, P=0.025\right.$ and $R^{2}=0.28, P=0.022$, respectively). $\mathbf{L}$ A negative correlation was found between average distance of OPCs from closest PVALB + cell and PNNs density $\left(R^{2}=0.36, P=0.024\right)$, suggesting that OPCs proximity with $P V A L B+$ cells could be associated with changes in PNN density. M Proximity of OPCs with PVALB+ cells was increased in DS-CA subjects ( $N=90$ OPCs, 5 subjects) compared to CTRLs ( $N=106$ OPCs, 6 subjects) and DS ( $N=73$ OPCs, 4 subjects) (one-way ANOVA: $F(2,266)=7.963, P=0.0004$, followed by Tukey's multiple comparison test). $\mathbf{N}$ Average densities of PDGFRA+ OPCs were not changed between $D S-C A(N=8)$, DS $(N=7)$, and CTRL $(N=6)$ groups (Kruskal-Wallis ANOVA, $\mathrm{H}(2,21)=4.67, P=0.095)$.

composition, and perhaps function, and that WFL-labeled PNNs may be biased towards specific neuronal types [32,33]. The use of additional markers should help decipher how ELA affects the remodeling of the ECM more broadly. It is also noteworthy that this analysis, by focusing on cortical layers IV-V, did not allow to clarify the possible layer specificity of PNN cellular distribution. Given the molecular, cellular, and connectivity heterogeneity in different cortical layers, it is possible that PNNs differentially interact with these different cell types, and that ELA may affect these interactions in a layer-specific manner.

One particularly noteworthy aspect of our results is the specific association between changes in PNNs and a history of CA. When comparing DS and controls for the expression of all PNN-related genes reported in our study (Fig. 2A), based on the snRNA-seq 
data generated by Nagy et al. [20] (Supplementary Tables 30 and 31 ), none showed differential expression between groups in OPCs. The fact that our FISH experiments did not show significantly enhanced expression of these markers in DS samples is therefore consistent both with results obtained by Nagy et al. [20] and with our own findings, indicating that PNNs are more abundant and mature specifically in samples from DS with a history of CA. Overall, this suggests that although transcriptomic changes in OPCs may be a strong feature of depressed suicides [20], PNNrelated changes are more specific to a history of CA. As such changes are absent in depressed suicides without a history of $C A$, our findings suggest possible vulnerability windows during which $\mathrm{PV}+$ cell function and PNN maturation are more susceptible to experience-dependent remodeling and adversity. If these changes may mediate some of the negative mental health outcomes or cognitive and emotional traits associated with CA in adulthood, they likely do not represent a hallmark of depression, in accordance with a recent post-mortem study finding no change in the density of PNNs in the PFC of depressed patients [34].

The search for possible mechanisms involved in the effects of ELA on PNN development is an unexplored field. PNN recruitment is likely orchestrated by a complex interplay between activitydependent autonomous pathways in parvalbumin neurons, with signals originating from different cell types involved in their assembly. As parvalbumin neurons have been shown to be particularly sensitive to stress and glucocorticoids, in particular early-life stress [35-39], we can speculate that elevated glucocorticoids in CA victims [40] can impact PV+ neuron function early-on. This could translate into increased GABA release following glucocorticoid receptor activation [38] and indirect increase in the recruitment of PNNs, which has been directly linked to PV+ neuron activity [41,42] and GABA levels [43]. A myriad of factors could however indirectly affect PV+ cells during this period of protracted maturation associated with childhood and adolescence, such as increased pro-inflammatory cytokine expression associated with ELA $[36,44]$ or changes in neurotrophic factor expression [45-47].

An interesting molecular candidate is the transcription factor OTX2, released non-locally by cells in the choroid plexus and acting as a major initiator of PNN development [48]. Recent evidence suggests a role of OTX2 in mediating vulnerability to early-life stress [49] and Murthy et al. [9] reported elevated expression of OTX2 in the choroid plexus following maternal separation and around PV+/PNNs + cells in the ventral dentate gyrus. Although the precise mechanisms involved in the effects of ELA on the release of OTX2 are not known, it is noteworthy that DNA methylation of the OTX2 gene in children has been shown to correlate with increased risk for depression as well as increased functional connectivity between the vmPFC and bilateral regions of the medial frontal cortex [50]. This highlights that beyond discrete changes in PV+ cell function, ELA could affect the release of distal cues by non-neuronal cells and contribute to extracellular matrix remodeling, thus affecting brain function and vulnerability to psychopathology.

As mentioned previously, although PNN development has been strongly linked to neuronal activity [3,41,51], PNN integrity and assembly are likely orchestrated by the complex integration by PV + neurons of cues originating from multiple cell types [52,53]. Accordingly, we found that the expression of genes encoding for the major canonical components of PNNs were strongly enriched in oligodendrocyte-lineage cells, in particular in OPCs, whereas PV + neurons barely expressed any of those components. Although our single-cell expression data originates from the dorsolateral PFC, this was validated in the vmPFC, thus decreasing the possibility that this pattern of enrichment is region-specific. This is also in accordance with previous literature in rats, albeit in the cerebellum, similarly showing that VCAN, PTPRZ1, and TNR are almost exclusively expressed in oligodendrocyte-lineage cells [54].
This is also consistent with more recent single-cell RNA-seq studies showing an enrichment of extracellular components, including VCAN and PTPRZ1, in OPCs [55-57].

The interplay between oligodendrocyte-lineage cells and PV+ neurons, in particular during developmental windows of plasticity, are being increasingly documented [58]. Interestingly, OPCs have been shown to be ontogenetically related to PV+ neurons [59]. OPCs also receive functional synaptic inputs from GABAergic interneurons, a connectivity that reaches its peak during early postnatal development along with the maturation of PNNs [6062]. It is therefore particularly tempting to speculate that OPC-PV neuron communication during critical windows of development may play a fundamental role in modeling cortical plasticity and the maturation of PNNs. This has never been addressed and we therefore approached this question by investigating how CA associates with changes in the expression of PNN canonical components specifically in OPCs, and how this correlates with the changes in PNN integrity observed in victims of CA. First, we found that OPC proximity to $\mathrm{PV}+$ neurons positively correlated with PNN densities, and that in victims of CA, OPCs tended to be more proximal to $\mathrm{PV}+$ neurons. As a proportion of OPCs are in direct physical contact with neuronal populations, and preferentially around GABAergic neurons $[27,63]$, we could speculate that CA associates with increased recruitment of OPCs around PV+ cells, thereby promoting the formation of PNNs around these cells. Caution is however needed in interpreting these correlational results that functionally relate the distance between OPCs and PV + cells to the recruitment of PNNs. The high percentage of PV+ cells enwrapped by PNNs in our data ( 65\%) and low percentage of PV + cells paired with an OPC $(\sim 3 \%$ based on [27], albeit in rodents) rather indicates that is unlikely that direct pairing of OPCs is necessary for the formation of PNNs. This question should therefore be further addressed with appropriate functional tools in cellular or animal models. Of important note, we did not address whether OPCs proximity with other cell types was changed, and how it could relate to changes in various cell populations. In addition, although we did not observe any change in overall OPCs densities between groups, a more refined layer-specific analysis of OPCs densities would be informative to rule out the influence of cell numbers on the physical proximity of these two cell types.

Second, we also found that the expression of canonical components of PNNs in OPCs was positively correlated with PNN densities and upregulated in victims of CA. This strongly suggests that CA has a durable impact on OPC molecular programs that may contribute to PNN development. Although we focused on VCAN, PTPRZ1, and TNR based on their high enrichment in OPCs, it would be important to describe more broadly how CA affects the transcriptional signature of PNNs. In particular, some of these components, although present in PNNs, are also found in other ECM compartments, such as the perinodal ECM and around excitatory cells, contributing to synaptic function [3]. Although we did not address this question, the impact of CA on OPC function may therefore more broadly affect ECM physiology and remodeling. It is also becoming increasingly clear that multiple populations with distinct functional or transcriptomic features are encompassed in OPCs $[64,65]$. While we can speculate that these subtypes may have distinct role in the remodeling of the ECM, we did not address this important question.

Our results, nonetheless, further strengthen previous reports by our group $[66,67]$ that CA has profound effects on oligodendrocyte-lineage cells, which may extend well beyond affecting myelination by contributing to the reprogramming of various aspects of brain plasticity.

How precisely OPC-PV communication contributes to the development of PNNs and impacts PV+ neuron functional integration and circuit dynamics certainly deserves further investigation using functional approaches. Inherent to our post- 
mortem design, a major limitation of this study lies in our inability to infer on the precise timing of these changes and whether dynamic remodeling of the ECM occurs in a protracted way. Given the correlational nature of our results, we cannot elaborate on the possible influence of recent and emotionally salient events on PNN remodeling, or possible state-dependent factors at the time of death. Several rodent studies have indeed reported that behavioral manipulations in adulthood, in particular learning and memory paradigms in which plasticity events are recruited, can affect PNN dynamics [68-71].

Although it is also tempting to infer that CA selectively affects PNN remodeling around PV+ neurons, caution is needed in this interpretation. As previously mentioned, WFL immunostaining may only label a specific fraction of PNNs [32,33], and the continuum between PNN components and other ECM compartments imply more refined approaches are necessary to fully understand how CA impacts ECM remodeling and the role of OPCs in this form of plasticity.

Another limitation is that our study only included very few female samples, given the much higher prevalence of suicide in males. Sex is known to moderate both the biological and the psychopathological effects of CA [72], and increasing evidence points towards a sexual dimorphism in the effects of stress, in particular early-life stress, on $\mathrm{PV}+$ neuron function and perhaps PNN development $[8,39,73]$. Future studies should therefore explore this aspect to further understand how ELA modifies trajectories of brain development.

Other limitations are inherent to post-mortem studies of psychiatric cohorts, such as the presence of medication in some subjects. Although this was the case for both depressed suicides groups, and that the observed changes seem specific to a history of CA, we cannot exclude interactive effects of medical treatments and life trajectories on our variables. Similarly, relatively long PMIs in our cohorts, although not statistically linked to changes in our variables, could potentially confound our results.

To conclude, our findings suggest that CA may lead to persistent patterns of maladaptive behaviors by reducing the neuroplasticity of cortical circuits through the enhancement of developmental OPC-mediated PNN formation. Future preclinical models should help determine whether changes in OPCs are causal in the increased recruitment of PNNs following CA or an indirect response following altered PNN dynamics. Likewise, the consequences of these molecular changes should be examined at the network level to determine their functional impact on intraand inter-regional communication.

\section{REFERENCES}

1. Teicher MH, Samson JA, Anderson CM, Ohashi K. The effects of childhood maltreatment on brain structure, function and connectivity. Nat Rev Neurosci. 2016;17:652-66.

2. Ferguson BR, Gao W-J. PV interneurons: critical regulators of E/I balance for prefrontal cortex-dependent behavior and psychiatric disorders. Front Neural Circuits. 2018;12:37.

3. Fawcett JW, Oohashi T, Pizzorusso T. The roles of perineuronal nets and the perinodal extracellular matrix in neuronal function. Nat. Rev. Neurosci. 2019. https://doi.org/10.1038/s41583-019-0196-3.

4. Sorg BA, Berretta S, Blacktop JM, Fawcett JW, Kitagawa H, Kwok JCF et al. Casting a wide net: role of perineuronal nets in neural plasticity. J Neurosci. 2016;36:11459-68.

5. Gogolla N, Caroni P, Lüthi A, Herry C. Perineuronal nets protect fear memories from erasure. Science. 2009;325:1258-61.

6. Thompson EH, Lensjø KK, Wigestrand MB, Malthe-Sørenssen A, Hafting T, Fyhn M. Removal of perineuronal nets disrupts recall of a remote fear memory. Proc Natl Acad Sci USA. 2018;115:607-12.

7. Shi W, Wei X, Wang X, Du S, Liu W, Song J et al. Perineuronal nets protect longterm memory by limiting activity-dependent inhibition from parvalbumin interneurons. Proc Natl Acad Sci USA. 2019. https://doi.org/10.1073/pnas.1902680116.

8. Bath K, Manzano-Nieves G, Goodwill H. Early life stress accelerates behavioral and neural maturation of the hippocampus in male mice. Horm Behav. 2016;82:64-71.
9. Murthy S, Kane GA, Katchur NJ, Lara Mejia PS, Obiofuma G, Buschman TJ, et al. Perineuronal nets, inhibitory interneurons, and anxiety-related ventral hippocampal neuronal oscillations are altered by early life adversity. Biol Psychiatry. 2019;85:1011-20.

10. Tanti A, Kim JJ, Wakid M, Davoli M-A, Turecki G, Mechawar N. Child abuse associates with an imbalance of oligodendrocyte-lineage cells in ventromedial prefrontal white matter. Mol Psychiatry. 2018;23:2018-28.

11. Colich N, Rosen M, Williams E, McLaughlin K. Biological aging in childhood and adolescence following experiences of threat and deprivation: a systematic review and meta-analysis. Psychol Bull. 2020;146:721-64.

12. Hiser J, Koenigs $M$. The multifaceted role of the ventromedial prefrontal cortex in emotion, decision making, social cognition, and psychopathology. Biol Psychiatry. 2018;83:638-47.

13. Teicher M, Samson J. Annual research review: enduring neurobiological effects of childhood abuse and neglect. J Child Psychol Psychiatry. 2016;57:241-66.

14. Lutz P-E, Tanti A, Gasecka A, Barnett-Burns S, Kim JJ, Zhou Y et al. Association of a history of child abuse with impaired myelination in the anterior cingulate cortex: convergent epigenetic, transcriptional, and morphological evidence. Am J Psychiatry. 2017: appi.ajp.2017.1.

15. Bifulco A, Brown GW, Harris TO. Childhood experience of care and abuse (CECA): a retrospective interview measure. J Child Psychol Psychiatry. 1994;35:1419-35.

16. Bifulco A, Brown GW, Lillie A, Jarvis J. Memories of childhood neglect and abuse: corroboration in a series of sisters. J Child Psychol Psychiatry. 1997;38:365-74.

17. Mai JK, Paxinos G, Voss T. Atlas of the human brain. Elsevier Acad. Press; 2008.

18. Schindelin J, Arganda-Carreras I, Frise E, Kaynig V, Longair M, Pietzsch T, et al. Fiji: an open-source platform for biological-image analysis. Nat Methods. 2012;9:676-82.

19. Bankhead $P$, Loughrey MB, Fernández JA, Dombrowski Y, McArt DG, Dunne PD, et al. QuPath: open source software for digital pathology image analysis. Sci Rep. 2017;7:1-7.

20. Nagy C, Maitra M, Tanti A, Suderman M, Théroux JF, Davoli MA, et al. Singlenucleus transcriptomics of the prefrontal cortex in major depressive disorder implicates oligodendrocyte precursor cells and excitatory neurons. Nat Neurosci. 2020;23:771-81.

21. Mauney S, Athanas K, Pantazopoulos H, Shaskan N, Passeri E, Berretta S, et al. Developmental pattern of perineuronal nets in the human prefrontal cortex and their deficit in schizophrenia. Biol Psychiatry. 2013;74:427-35.

22. Pantazopoulos $\mathrm{H}$, Lange $\mathrm{N}$, Hassinger L, Berretta S. Subpopulations of neurons expressing parvalbumin in the human amygdala. J Comp Neurol. 2006;496:706-22

23. Morikawa S, Ikegaya $\mathrm{Y}$, Narita $M$, Tamura $H$. Activation of perineuronal netexpressing excitatory neurons during associative memory encoding and retrieval. Sci Rep. 2017;7. https://doi.org/10.1038/SREP46024.

24. Carstens K, Phillips M, Pozzo-Miller L, Weinberg R, Dudek S. Perineuronal nets suppress plasticity of excitatory synapses on CA2 pyramidal neurons. J Neurosci. 2016;36:6312-20.

25. Enwright JF, Sanapala S, Foglio A, Berry R, Fish KN, Lewis DA. Reduced labeling of parvalbumin neurons and perineuronal nets in the dorsolateral prefrontal cortex of subjects with schizophrenia. Neuropsychopharmacology. 2016;41:2206-14.

26. Perlman K, Couturier CP, Yaqubi M, Tanti A, Cui Q, Pernin F, et al. Developmental trajectory of oligodendrocyte progenitor cells in the human brain revealed by single cell RNA sequencing. Glia. 2020;68:1291-303.

27. Boulanger JJ, Messier C. Oligodendrocyte progenitor cells are paired with GABA neurons in the mouse dorsal cortex: unbiased stereological analysis. Neuroscience. 2017;362:127-40.

28. Guadagno A, Verlezza S, Long $H$, Wong TP, Walker CD. It is all in the right amygdala: increased synaptic plasticity and perineuronal nets in male, but not female, juvenile rat pups after exposure to early-life stress. J Neurosci. 2020;40:8276-91.

29. Gildawie KR, Honeycutt JA, Brenhouse HC. Region-specific effects of maternal separation on perineuronal net and parvalbumin-expressing interneuron formation in male and female rats. Neuroscience. 2019. https://doi.org/10.1016/j. neuroscience.2019.12.010.

30. Ueno H, Suemitsu S, Murakami S, Kitamura N, Wani K, Matsumoto $Y$, et al. Juvenile stress induces behavioral change and affects perineuronal net formation in juvenile mice. BMC Neurosci. 2018;19:1-21.

31. Lensjø K, Christensen A, Tennøe S, Fyhn M, Hafting T. Differential expression and cell-type specificity of perineuronal nets in hippocampus, medial entorhinal cortex, and visual cortex examined in the rat and mouse. eNeuro. 2017;4. https:// doi.org/10.1523/ENEURO.0379-16.2017.

32. Matthews R, Kelly G, Zerillo C, Gray G, Tiemeyer M, Hockfield S. Aggrecan glycoforms contribute to the molecular heterogeneity of perineuronal nets. J Neurosci. 2002;22:7536-47.

33. Galtrey CM, Kwok JCF, Carulli D, Rhodes KE, Fawcett JW. Distribution and synthesis of extracellular matrix proteoglycans, hyaluronan, link proteins and tenascin-R in the rat spinal cord. Eur J Neurosci. 2008;27:1373-90. 
34. Alcaide J, Guirado R, Crespo C, Blasco-lbáñez JM, Varea E, Sanjuan J, et al. Alterations of perineuronal nets in the dorsolateral prefrontal cortex of neuropsychiatric patients. Int J Bipolar Disord. 2019;7:24.

35. Helmeke C, Ovtscharoff W, Poeggel G, Braun K. Imbalance of immunohistochemically characterized interneuron populations in the adolescent and adult rodent medial prefrontal cortex after repeated exposure to neonatal separation stress. Neuroscience. 2008;152:18-28.

36. Grassi-Oliveira R, Honeycutt JA, Holland FH, Ganguly P, Brenhouse HC. Cognitive impairment effects of early life stress in adolescents can be predicted with early biomarkers: Impacts of sex, experience, and cytokines. Psychoneuroendocrinology. 2016;71:19-30.

37. Uchida T, Furukawa T, Iwata S, Yanagawa Y, Fukuda A. Selective loss of parvalbumin-positive GABAergic interneurons in the cerebral cortex of maternally stressed Gad1-heterozygous mouse offspring. Transl Psychiatry. 2014;4:e371.

38. Hu W, Zhang M, Czéh B, Flügge G, Zhang W. Stress impairs GABAergic network function in the hippocampus by activating nongenomic glucocorticoid receptors and affecting the integrity of the parvalbumin-expressing neuronal network. Neuropsychopharmacology. 2010. https://doi.org/10.1038/npp.2010.31.

39. Holland FH, Ganguly P, Potter DN, Chartoff EH, Brenhouse HC. Early life stress disrupts social behavior and prefrontal cortex parvalbumin interneurons at an earlier time-point in females than in males. Neurosci Lett. 2014;566:131-6.

40. Heim C, Mletzko T, Purselle D, Musselman DL, Nemeroff CB. The dexamethasone/ corticotropin-releasing factor test in men with major depression: role of childhood trauma. Biol Psychiatry. 2008;63:398-405.

41. Devienne G, Picaud S, Cohen I, Piquet J, Tricoire L, Testa D et al. Regulation of perineuronal nets in the adult cortex by the electrical activity of parvalbumin interneurons. J Neurosci. 2019;41:5779-90. https://doi.org/10.1101/671719.

42. Cisneros-Franco JM, De Villers-Sidani É. Reactivation of critical period plasticity in adult auditory cortex through chemogenetic silencing of parvalbumin-positive interneurons. Proc Natl Acad Sci USA. 2019. https://doi.org/10.1073/pnas.1913227117.

43. Deidda G, Allegra M, Cerri C, Naskar S, Bony G, Zunino G et al. Early depolarizing GABA controls critical-period plasticity in the rat visual cortex. Nat Neurosci. 2015. https://doi.org/10.1038/nn.3890.

44. Heim C, Newport DJ, Mletzko T, Miller AH, Nemeroff CB. The link between childhood trauma and depression: Insights from HPA axis studies in humans. Psychoneuroendocrinology. 2008;33:693-710.

45. Huang ZJ, Kirkwood A, Pizzorusso T, Porciatti V, Morales B, Bear MF et al. BDNF regulates the maturation of inhibition and the critical period of plasticity in mouse visual cortex. Cell. 1999. https://doi.org/10.1016/S0092-8674(00)81509-3.

46. Cirulli F, Francia N, Berry A, Aloe L, Alleva E, Suomi SJ. Early life stress as a risk factor for mental health: Role of neurotrophins from rodents to non-human primates. Neurosci. Biobehav. Rev. 2009. https://doi.org/10.1016/j.neubiorev.2008.09.001.

47. Begum MR, Sng JCG. Molecular mechanisms of experience-dependent maturation in cortical GABAergic inhibition. J. Neurochem. 2017. https://doi.org/10.1111/ jnc. 14103.

48. Beurdeley M, Spatazza J, Lee HHC, Sugiyama S, Bernard C, Di Nardo AA, et al. Otx2 binding to perineuronal nets persistently regulates plasticity in the mature visual cortex. J Neurosci. 2012;32:9429-37.

49. Peña CJ, Kronman HG, Walker DM, Cates HM, Bagot RC, Purushothaman I et al. Early life stress confers lifelong stress susceptibility in mice via ventral tegmental area OTX2. Science. 2017. https://doi.org/10.1126/science.aan4491.

50. Kaufman J, Wymbs NF, Montalvo-Ortiz JL, Orr C, Albaugh MD, Althoff R et al. Methylation in OTX2 and related genes, maltreatment, and depression in children. Neuropsychopharmacology. 2018. https://doi.org/10.1038/s41386-018-0157-y.

51. Dityatev A, Brückner G, Dityateva G, Grosche J, Kleene R, Schachner M. Activitydependent formation and functions of chondroitin sulfate-rich extracellular matrix of perineuronal nets. Dev Neurobiol. 2007;67:570-88.

52. Simard S, Coppola G, Rudyk CA, Hayley S, McQuaid RJ, Salmaso N. Profiling changes in cortical astroglial cells following chronic stress. Neuropsychopharmacology. 2018. https://doi.org/10.1038/s41386-018-0105-x.

53. Song I, Dityatev A. Crosstalk between glia, extracellular matrix and neurons. Brain Res. Bull. 2018. https://doi.org/10.1016/j.brainresbull.2017.03.003.

54. Carulli D, Rhodes KE, Brown DJ, Bonnert TP, Pollack SJ, Oliver K, et al. Composition of perineuronal nets in the adult rat cerebellum and the cellular origin of their components. J Comp Neurol. 2006;494:559-77.

55. Velmeshev D, Schirmer L, Jung D, Haeussler M, Perez $Y$, Mayer $S$, et al. Single-cell genomics identifies cell type-specific molecular changes in autism. Science. 2019;364:685-9.

56. Kumar MP, Du J, Lagoudas G, Jiao Y, Sawyer A, Drummond DC, et al. Analysis of single-cell RNA-seq identifies cell-cell communication associated with tumor characteristics. Cell Rep. 2018;25:1458-.e4.

57. Habib N, Avraham-Davidi I, Basu A, Burks T, Shekhar K, Hofree M, et al. Massively parallel single-nucleus RNA-seq with DroNc-seq. Nat Methods. 2017. https://doi. org/10.1038/nmeth.4407
58. Yang SM, Michel K, Jokhi V, Nedivi E, Arlotta P. Neuron class-specific responses govern adaptive myelin remodeling in the neocortex. Science. 2020;370. https:// doi.org/10.1126/SCIENCE.ABD2109.

59. Orduz D, Benamer N, Ortolani D, Coppola E, Vigier L, Pierani A, et al. Developmental cell death regulates lineage-related interneuron-oligodendroglia functional clusters and oligodendrocyte homeostasis. Nat Commun. 2019;10:4249.

60. Orduz D, Maldonado PP, Balia M, Vélez-Fort M, de Sars V, Yanagawa $Y$, et al. Interneurons and oligodendrocyte progenitors form a structured synaptic network in the developing neocortex. Elife. 2015;2015:1-53.

61. Lin SC, Bergles DE. Synaptic signaling between GABAergic interneurons and oligodendrocyte precursor cells in the hippocampus. Nat Neurosci. 2004. https:// doi.org/10.1038/nn1162.

62. Vélez-Fort M, Maldonado PP, Butt AM, Audinat E, Angulo MC. Postnatal switch from synaptic to extrasynaptic transmission between interneurons and NG2 cells. J Neurosci. 2010. https://doi.org/10.1523/JNEUROSCI.0238-10.2010.

63. Butt AM, Hamilton N, Hubbard P, Pugh M, Ibrahim M. Synantocytes: the fifth element. J Anat. 2005;207:695-706.

64. Bribián A, Medina-Rodríguez EM, Josa-Prado F, García-Álvarez I, Machín-Díaz I, Esteban PF, et al. Functional heterogeneity of mouse and human brain OPCs: relevance for preclinical studies in multiple sclerosis. J Clin Med. 2020;9:1681.

65. Spitzer SO, Sitnikov S, Kamen Y, Evans KA, Kronenberg-Versteeg D, Dietmann S, et al. Oligodendrocyte progenitor cells become regionally diverse and heterogeneous with age. Neuron. 2019;101:459-.e5.

66. Lutz P-E, Tanti A, Gasecka A, Barnett-Burns S, Kim JJ, Zhou Y et al. Association of a history of child abuse with impaired myelination in the anterior cingulate cortex: convergent epigenetic, transcriptional, and morphological evidence. Am J Psychiatry. 2017:appiajp201716111286.

67. Tanti A, Kim JJ, Wakid M, Davoli M-A, Turecki G, Mechawar N. Child abuse associates with an imbalance of oligodendrocyte-lineage cells in ventromedial prefrontal white matter. Mol Psychiatry. 2018;23:2018-28.

68. Slaker M, Jorgensen E, Hegarty D, Liu X, Kong Y, Zhang F et al. Cocaine exposure modulates perineuronal nets and synaptic excitability of fast-spiking interneurons in the medial prefrontal cortex. eNeuro. 2018;5. https://doi.org/10.1523/ ENEURO.0221-18.2018.

69. Banerjee SB, Gutzeit VA, Baman J, Aoued HS, Doshi NK, Liu RC, et al. Perineuronal nets in the adult sensory cortex are necessary for fear learning. Neuron. 2017;95:169-.e3.

70. Pintér A, Hevesi Z, Zahola P, Alpár A, Hanics J. Chondroitin sulfate proteoglycan-5 forms perisynaptic matrix assemblies in the adult rat cortex. Cell Signal. 2020;74. https://doi.org/10.1016/J.CELLSIG.2020.109710.

71. Pantazopoulos H, Gisabella B, Rexrode L, Benefield D, Yildiz E, Seltzer P, et al. Circadian rhythms of perineuronal net composition. eNeuro. 2020;7:1-21.

72. Wellman CL, Bangasser DA, Bollinger JL, Coutellier L, Logrip ML, Moench KM, et al. Sex differences in risk and resilience: stress effects on the neural substrates of emotion and motivation. J Neurosci. 2018;38:9423-32.

73. Gildawie KR, Honeycutt JA, Brenhouse HC. Region-specific effects of maternal separation on perineuronal net and parvalbumin-expressing interneuron formation in male and female rats. Neuroscience. 2020. https://doi.org/10.1016/j. neuroscience.2019.12.010.

\section{AUTHOR CONTRIBUTIONS}

AT and NM conceived the study. GT participated in the acquisition and clinical characterization of the brain samples. AT, CB, FD, MAD, CC, RM, and AM contributed to immunohistological experiments. $\mathrm{AT}, \mathrm{CN}, \mathrm{MM}$, and KP generated and analyzed the snRNA-seq data set. AT, CB, FC, and ST performed the in situ hybridization experiments. $\mathrm{AT}, \mathrm{CB}$, and NM prepared the manuscript, and all authors contributed to and approved its final version.

\section{FUNDING}

This work was funded by a CIHR Project grant (PJT-173287) to NM. AT was supported by fellowships from the FRQ-S and Toronto Dominion, and an American Foundation for Suicide Prevention (AFSP) Young Investigator Innovation Grant (YIG-0-146-17). C. Belliveau holds an FRQ-S doctoral scholarship. The Molecular and Cellular Microscopy Platform and the Douglas-Bell Canada Brain Bank (DBCBB) are partly funded by Healthy Brains for Healthy Lives (CFREF) and Brain Canada platform grants to GT and NM. The DBCBB is also funded by the Réseau Québécois sur le suicide, le troubles de I'humeur et les troubles associés (FRQ-S).

\section{COMPETING INTERESTS}

The authors declare no competing interest. 


\section{ADDITIONAL INFORMATION}

Supplementary information The online version contains supplementary material available at https://doi.org/10.1038/s41380-021-01372-y.

Correspondence and requests for materials should be addressed to Arnaud Tanti or Naguib Mechawar.

Reprints and permission information is available at http://www.nature.com/ reprints

Publisher's note Springer Nature remains neutral with regard to jurisdictional claims in published maps and institutional affiliations.
Open Access This article is licensed under a Creative Commons Attribution 4.0 International License, which permits use, sharing, adaptation, distribution and reproduction in any medium or format, as long as you give appropriate credit to the original author(s) and the source, provide a link to the Creative Commons license, and indicate if changes were made. The images or other third party material in this article are included in the article's Creative Commons license, unless indicated otherwise in a credit line to the material. If material is not included in the article's Creative Commons license and your intended use is not permitted by statutory regulation or exceeds the permitted use, you will need to obtain permission directly from the copyright holder. To view a copy of this license, visit http://creativecommons.org/licenses/by/4.0/.

(c) The Author(s) 2021 